\title{
LIVER IMPAIRMENT AS A PREDICTIVE FACTOR FOR MORTALITY RATE OF COVID-19 PATIENTS
}

By

\author{
Ashraf Abd El-Aty El-Shenawy Emara ${ }^{1,3}$, Amin Mahmoud Amin \\ Hegazy $^{1}$, Ahmed Mohamed Abu Hassan ${ }^{1}$ and Ahmed Mohamed El-Deeb ${ }^{2}$ \\ ${ }^{1}$ Department of Internal Medicine, Faculty of Medicine, Al-Azhar University, Cairo, Egypt \\ ${ }^{2}$ Department of Diagnostic Radiology, Faculty of Medicine, Al-Azhar University, Cairo, \\ Egypt \\ ${ }^{3}$ Department of Medical Physiology, Faculty of Medicine, Al-Azhar University, Cairo, \\ Egypt \\ Corresponding author: Ashraf Abd El-Aty El-Shenawy Emara, \\ Mobile: 01003139935, E-mail: ashrfemara13@gmail.com
}

\begin{abstract}
Background: In the current COVID-19 pandemic, which is caused by severe acute respiratory syndromecoronavirus-2 (SARS-CoV2), disease diagnosis is essential for optimal management, timely isolation of infected cases in order to prevent further spread and is associated with significant morbidity and mortality burden.
\end{abstract}

Objective: To assess the liver impairment as a predictive factor for mortality rate of COVID -19 patients.

Patients and Methods: The prospective study included 100 patients with Covid-19 infection who were admitted to Al-Hussein and Bab Al-Sha'aria University Hospitals. The study was during the period from 1st of February 2021 to 31th of May 2021 and divided in to five groups according to liver functions tests. Presenting clinical manifestations, laboratory findings, radiological findings and mortality rate were recorded from electronic medical records. Diagnoses of COVID-19 patient were done according to criteria of ministry of health and population- Egypt.

Results: The results revealed that the mean of platelets reaching to a minimum mean value at group E and the mean of the neutrophil /lymphocyte ratio reaching to a maximum mean value at Group E, while the lymphocyte /monocyte ratio reaching to a minimum mean value at Group E. There were significant difference among all groups regarding ALT, AST, total bilirubin, direct bilirubin and serum Albumin with P value $=0.001$ in all parameters. Also there were significant decreases of serum albumin in groups $\mathrm{C}, \mathrm{D}$ and $\mathrm{E}$ when compared with group $\mathrm{A}$, and there were significant increases of total bilirubin, direct bilirubin mean values in group $\mathrm{E}$ when compared with group A. There were significant increase of prothrombin time (PT) and INR in groups $\mathrm{E}$ when compared with group A. The results showed a significant difference (p-value $<0.05$ ) between recovered and died cases for each group with higher mortality rate at group D ( 7 cases with a percent of $30.4 \%$ ), then at group E ( 3 cases with a percent of $25 \%$ ), and there were $88 \%$ recovered and $12 \%$ died in all studied COVID-19 patients. The results revealed that the higher mortality rates at group D and E between patients having chest CT scoring 4 and 5 .

Conclusion: Patients with accompanying chronic liver diseases are predisposed to developing a more severe course of COVID-19 and increase the mortality rate, but on the other hand, a more complicated presentation of SARS-CoV-2 infection increases the risk of liver failure.

Keywords: COVID-19, liver impairment chronic liver diseases, mortality rate. 


\section{INTRODUCTION}

Accumulated data suggest that more than one-third of patients hospitalized due to SARS-CoV-2 infection might have impaired liver function. An increase in aspartate transaminase (AST) and alanine transaminase (ALT) activity, especially in men, results in a severe course of COVID19. In general, a higher level of ALT, thrombocytopenia and hypoalbuminemia are indices of increased mortality in COVID-19 patients. Moreover, hypoalbuminemia is recognized as an independent marker of severe SARSCoV-2 infection, poor prognosis and higher mortality (Gholizadeh et al., 2020 and Liu et al., 2020-b), but the change in albumin does not parallel the severity of hepatocellular injury in COVID-19 (Zhang et al., 2020). This suggests that there may be mechanisms other than a hepatocellular injury that explains the profound hypoalbuminemia seen in COVID-19. One of the possible mechanisms is the intense systemic inflammation being reported in severe COVID-19 (Qin et al., 2020). Hypoalbuminemia is common in many inflammatory diseases because increased capillary permeability can result in the escape of albumin to the interstitial space (Soeters et al., 2019).

For diagnosis, health specialists use the Reverse Transcription Polymerase Chain Reaction (RT-PCR) test for the detection of the nucleic acid of the SARS-CoV-2 in the respiratory specimens (such as oropharyngeal swabs or nasopharyngeal sampling) (Liu et al., 2020-c). Chest CT imaging may also be helpful for diagnosis in individuals where there is a high suspicion of infection based on symptoms and risk factors; however, guidelines do not recommend using CT imaging for routine screening (Salehi et al., 2020).

Recommended measures to prevent infection include frequent hand washing, maintaining physical distance from others (especially from those with symptoms), quarantine (especially for those with symptoms), covering coughs, and keeping.

The present study aimed to understand the impairment of the liver as a predictive factor for mortality rate of Covid-19 patients.

\section{PATIENTS AND METHODS}

This prospective study included 100 patients with Covid-19 infection who were admitted to Al-Hussein and Bab AlSha'aria University Hospitals. The cases were collected during the period from $1 \mathrm{st}$ of February 2021 to 31th of May 2020. All procedures followed Al-Azhar University Ethical Committee regulations. Written consents were taken from all patients before collecting any information or starting any procedure. Patients were divided in to five groups according to liver functions tests as follow:

- Group A included COVID-19 patients who were medically free and showed normal liver functions tests after affection.

- Group B included COVID-19 patients who known to have chronic illness and showed normal liver functions tests after affection.

- Group C included COVID-19 patients who were medically free and showed disturbed liver functions tests after affection. 


\section{LIVER IMPAIRMENT AS A PREDICTIVE FACTOR FOR MORTALITY...}

- Group D included COVID-19 patients who known to have chronic illness and showed disturbed liver functions tests after affection.

- Group E included COVID-19 patients who had compensated liver diseases and showed disturbed liver functions tests after affection.

Inclusion criteria: Patients of all ages, both genders, positive results confirmed by standard SARS-CoV-2 RT-PCR.

Exclusion criteria: - ve SARS-CoV-2 RT-PCR test.

All patients were subjected to the following procedures: 1- Full history including demographic characteristics. 2General physical and systemic examination. 3- Routine investigations including: -Liver function tests (ALT, AST, total bilirubin, serum albumin, prothrombin time and INR) and renal function tests (serum creatinine and blood urea). -Complete blood count (HGB, RBCs count, platelet count, WBCs count, lymphocyte-monocyte ratio and neutrophil-lymphocyte ratio). 4Radiological investigations: CT Chest for all patients. On CT chest, each of the 5 lung lobes was visually scored from 0 to 5 as follows: 0 , no involvement; $1,<5 \%$ involvement; $2,5 \%-25 \%$ involvement; 3 , $26 \%-50 \%$ involvement; $4,51 \%-75 \%$ involvement; and 5,>75\% involvement (Pan et al., 2020). 5- Diagnosis of
COVID-19 patient according to criteria of ministry of health and population-Egypt. 6- All patients received medical treatment according to protocols of ministry of health and population Egypt.

\section{Statistical Analysis:}

The data were entered, coded and processed on computer using Statistical Packaged for the Social Science (IBM SPSS version 22, 2013). The level $\mathrm{P} \leq$ 0.05 was considered the cut-off value for significance. Description of quantitative variables was as mean, SD and range. Description of qualitative variables was as number and percentage. Chi-Square test $\chi^{2}$ was used to compare qualitative variables between groups. Kruskal-Wallis test was used for abnormally quantitative variables, to comparison among more than two groups. Mann Whitney U test was used for abnormally quantitative variables, to compare between two studied groups. Pearson correlation coefficient was used for measuring the strength and direction of a linear relationship between two variables. Repeated measures ANOVA were used for comparison among more than two means in quantitative data. In case of significance, least significant difference (LSD) test was performed to detect pairs of groups significantly different. 


\section{RESULTS}

The mean of platelets reaching to a minimum mean value of $183.17 \pm 118.45$ at group $\mathrm{E}$ (Table 1).

Table (1): Means and standard deviations of Age and blood cells and comparison between all groups

\begin{tabular}{|c|c|c|c|c|c|c|c|c|c|c|c|c|}
\hline \multicolumn{2}{|c|}{$\begin{array}{l}\text { Parameters } \\
\text { Variables }\end{array}$} & \multicolumn{3}{|c|}{ Range } & \multirow{2}{*}{$\begin{array}{r}\text { Mean } \\
47.80\end{array}$} & \multirow[t]{2}{*}{ \pm} & \multirow{2}{*}{$\begin{array}{c}\text { S. D } \\
20.05\end{array}$} & \begin{tabular}{|c|} 
p. \\
value
\end{tabular} & \multicolumn{4}{|c|}{ Post Hock test (LSD) } \\
\hline \multirow{5}{*}{ Age } & Group A & 20 & - & 80 & & & & \multirow{5}{*}{0.011} & $\mathrm{P} 1$ & 0.018 & P6 & 0.774 \\
\hline & Group B & 22 & - & 97 & 59.79 & \pm & 16.78 & & $\mathrm{P} 2$ & 0.955 & P7 & 0.447 \\
\hline & Group C & 20 & - & 66 & 48.12 & \pm & 14.10 & & $\mathrm{P} 3$ & 0.015 & $\mathrm{P} 8$ & 0.013 \\
\hline & Group D & 25 & - & 87 & 61.04 & \pm & 15.90 & & $\mathrm{P} 4$ & 0.011 & P9 & 0.010 \\
\hline & Group E & 44 & - & 76 & 63.92 & \pm & 9.61 & & $\mathrm{P} 5$ & 0.017 & P10 & 0.616 \\
\hline \multirow{5}{*}{ HGB } & Group A & 9.5 & - & 16 & 12.75 & \pm & 2.26 & \multirow{5}{*}{0.048} & $\mathrm{P} 1$ & 0.148 & P6 & 0.612 \\
\hline & Group B & 5.4 & - & 16.3 & 11.62 & \pm & 2.46 & & $\mathrm{P} 2$ & 0.449 & P7 & 0.345 \\
\hline & Group C & 8.4 & - & 17.8 & 13.41 & \pm & 2.77 & & $\mathrm{P} 3$ & 0.343 & P8 & 0.070 \\
\hline & Group D & 6.1 & - & 15.3 & 11.97 & \pm & 2.25 & & $\mathrm{P} 4$ & 0.048 & P9 & 0.001 \\
\hline & Group E & 5.7 & - & 15.6 & 10.83 & \pm & 2.70 & & P5 & 0.017 & P10 & 0.201 \\
\hline \multirow{5}{*}{ Platelets } & Group A & 109 & - & 462 & 279.73 & \pm & 97.91 & \multirow{5}{*}{0.201} & $\mathrm{P} 1$ & 0.601 & P6 & 0.392 \\
\hline & Group B & 58 & - & 521 & 260.85 & \pm & 113.62 & & $\mathrm{P} 2$ & 0.813 & P7 & 0.067 \\
\hline & Group C & 138 & - & 584 & 289.47 & \pm & 121.61 & & P3 & 0.548 & P8 & 0.376 \\
\hline & Group D & 15 & - & 454 & 256.57 & \pm & 123.26 & & $\mathrm{P} 4$ & 0.054 & P9 & 0.052 \\
\hline & Group E & 45 & - & 365 & 183.17 & \pm & 118.45 & & $\mathrm{P} 5$ & 0.410 & $\mathrm{P} 10$ & 0.078 \\
\hline \multirow{5}{*}{ WBCs } & Group A & 3 & - & 12.4 & 8.00 & \pm & 3.38 & \multirow{5}{*}{0.149} & $\mathrm{P} 1$ & 0.874 & P6 & 0.052 \\
\hline & Group B & 2.64 & - & 18.24 & 8.44 & \pm & 4.16 & & $\mathrm{P} 2$ & 0.124 & P7 & 0.352 \\
\hline & Group C & 3.1 & - & 69 & 12.85 & \pm & 15.03 & & P3 & 0.058 & P8 & 0.723 \\
\hline & Group D & 2.5 & - & 49 & 13.85 & \pm & 11.18 & & $\mathrm{P} 4$ & 0.348 & P9 & 0.625 \\
\hline & Group E & 3.5 & - & 24.2 & 11.22 & \pm & 5.30 & & P5 & 0.097 & P10 & 0.404 \\
\hline
\end{tabular}

P1: Group A \& Group B. P2: Group A \& Group C. P3: Group A \& Group D.

P4: Group A \& Group E. P5: Group B \& Group C. P6: Group B \& Group D.

P7: Group B \& Group E. P8: Group C \& Group D. P9: Group C \& Group E.

P10: Group D \& Group E. SD: Standard deviation. 
The mean of the neutrophil /lymphocyte ratio reaching to a maximum mean value of $12.21 \pm 8.71$ at group $\mathrm{E}$

while the lymphocyte /monocyte ratio reaching to a minimum mean value of $2.13 \pm 1.80$ at group E (Table 2).

Table (2): Means and standard deviations of some variables of differential leucocytic count and comparison between all groups.

\begin{tabular}{|c|c|c|c|c|c|c|c|c|}
\hline \multicolumn{2}{|c|}{$\begin{array}{ll}\text { Variables } & \text { Parameters } \\
\end{array}$} & \multicolumn{3}{|c|}{ Range } & \multirow{2}{*}{$\begin{array}{c}\text { Mean } \\
7.94\end{array}$} & \multirow{2}{*}{$\begin{array}{l} \pm \\
\pm\end{array}$} & \multirow{2}{*}{$\begin{array}{l}\text { S. D } \\
9.75\end{array}$} & \multirow[t]{2}{*}{ p. value } \\
\hline \multirow{5}{*}{$\begin{array}{l}\text { Neutrophil / } \\
\text { lymphocyte \% }\end{array}$} & Group A & 1.35 & - & 39.46 & & & & \\
\hline & Group B & 0.71 & - & 22.75 & 7.66 & \pm & 4.81 & \multirow{4}{*}{0.629} \\
\hline & Group C & 1.03 & - & 26.34 & 9.23 & \pm & 7.13 & \\
\hline & Group D & 2.01 & - & 31 & 11.37 & \pm & 10.04 & \\
\hline & Group E & 1.47 & - & 31 & 12.21 & \pm & 8.71 & \\
\hline \multirow{5}{*}{$\begin{array}{c}\text { Lymphocyte / } \\
\text { monocyte } \\
\%\end{array}$} & Group A & 0.74 & - & 10 & 4.71 & \pm & 2.41 & \multirow{5}{*}{0.296} \\
\hline & Group B & 0.48 & - & 23.13 & 3.15 & \pm & 4.02 & \\
\hline & Group C & 0.7 & - & 5.37 & 3.02 & \pm & 1.73 & \\
\hline & Group D & 0.75 & - & 31.21 & 4.69 & \pm & 6.76 & \\
\hline & Group E & 0.63 & - & 6.66 & 2.13 & \pm & 1.80 & \\
\hline
\end{tabular}

SD: Standard deviation.

Kruskal-Wallis test showed significant difference among all groups regarding the serum creatinine and blood urea with $\mathrm{P}$ value $<0.05$ in both serum creatinine and blood urea (Table 3).

Table (3): Means and standard deviations of some variables of kidney functions tests and comparison between all groups

\begin{tabular}{|c|c|c|c|c|c|c|c|c|c|c|c|c|}
\hline \multicolumn{2}{|c|}{$\begin{array}{ll} & \text { Parameters } \\
\text { Variables } & \end{array}$} & \multicolumn{3}{|c|}{ Range } & \multirow{2}{*}{$\begin{array}{c}\text { Mean } \\
0.96 \\
\end{array}$} & \multirow{2}{*}{$\begin{array}{l} \pm \\
\pm\end{array}$} & \multirow{2}{*}{$\begin{array}{l}\text { S. D } \\
0.26\end{array}$} & $\begin{array}{c}\text { p. } \\
\text { value }\end{array}$ & \multicolumn{4}{|c|}{ Post Hock test (LSD) } \\
\hline \multirow{5}{*}{$\begin{array}{c}\text { Serum } \\
\text { Creatinine }\end{array}$} & Group A & 0.5 & - & 1.6 & & & & \multirow{5}{*}{0.006} & P1 & 0.643 & P6 & 0.001 \\
\hline & Group B & 0.3 & - & 5.3 & 1.18 & \pm & 0.93 & & $\mathrm{P} 2$ & 0.969 & P7 & 0.887 \\
\hline & Group C & 0.3 & - & 1.8 & 0.98 & \pm & 0.38 & & P3 & 0.001 & P8 & 0.001 \\
\hline & Group D & 0.6 & - & 11.1 & 2.81 & \pm & 2.86 & & $\mathrm{P} 4$ & 0.619 & P9 & 0.636 \\
\hline & Group E & 0.7 & - & 1.9 & 1.25 & \pm & 0.40 & & P5 & 0.661 & P10 & 0.004 \\
\hline \multirow{5}{*}{$\begin{array}{l}\text { Blood } \\
\text { Urea }\end{array}$} & Group A & 16 & - & 88 & 41.16 & \pm & 19.54 & \multirow{5}{*}{0.003} & P1 & 0.177 & P6 & 0.001 \\
\hline & Group B & 16 & - & 232 & 67.24 & \pm & 54.21 & & $\mathrm{P} 2$ & 1.0 & P7 & 0.764 \\
\hline & Group C & 8 & - & 86 & 41.16 & \pm & 20.45 & & P3 & 0.001 & P8 & 0.001 \\
\hline & Group D & 24.3 & - & 451 & 122.95 & \pm & 105.92 & & $\mathrm{P} 4$ & 0.408 & P9 & 0.395 \\
\hline & Group E & 30 & - & 117 & 61.00 & \pm & 26.69 & & P5 & 0.160 & $\mathrm{P} 10$ & 0.006 \\
\hline
\end{tabular}

P1: Group A \& Group B. P2: Group A \& Group C. P3: Group A \& Group D.

P4: Group A \& Group E. P5: Group B \& Group C. P6: Group B \& Group D.

P7: Group B \& Group E. P8: Group C \& Group D. P9: Group C \& Group E.

P10: Group D \& Group E. SD: Standard deviation. 
The mean values of ALT in groups C, $D$ and $E$ were $98.12 \pm 68.21 \& 69.39 \pm 48.49$ and $59.33 \pm 30.39$ respectively. The mean values of AST in groups C, D and E were $78.76 \pm 51.97 \quad \& \quad 81.93 \pm 69.19$ and $77.17 \pm 51.51$ respectively, and the mean values of serum albumin in groups $C, D$ and $\mathrm{E}$ were $3.70 \pm 0.62 \& 3.32 \pm 0.68$ and $2.47 \pm 0.78$ respectively. There were significant decreases of serum albumin in groups $\mathrm{C}, \mathrm{D}$ and $\mathrm{E}$ when compared with group $\mathrm{A}$ with $\mathrm{P}$ value $<0.05$. Also, there were significant increases of total bilirubin, direct bilirubin mean values in group E when compared with Group A with $\mathrm{P}$ value $<0.05$. Kruskal-Wallis test showed a significant difference among all groups regarding the ALT, AST, total bilirubin, direct bilirubin and serum albumin with $\mathrm{P}$ value $=0.001$ in all parameters (Table 4).

Table (4): Means and standard deviations of some variables of liver functions tests and comparison between all groups

\begin{tabular}{|c|c|c|c|c|c|c|c|c|c|c|c|c|}
\hline \multicolumn{2}{|c|}{$\begin{array}{l}\text { Parameters } \\
\text { Variables }\end{array}$} & \multicolumn{3}{|c|}{ Range } & \multirow{2}{*}{$\begin{array}{l}\text { Mean } \\
21.33 \\
\end{array}$} & \multirow{2}{*}{$\begin{array}{l} \pm \\
\pm\end{array}$} & \multirow{2}{*}{$\begin{array}{l}\text { S. D } \\
10.86\end{array}$} & $\begin{array}{c}\text { p. } \\
\text { value }\end{array}$ & \multicolumn{4}{|c|}{ Post Hock test (LSD) } \\
\hline \multirow{5}{*}{ ALT } & Group A & 6 & - & 38 & & & & \multirow{5}{*}{0.001} & P1 & 0.958 & P6 & 0.001 \\
\hline & Group B & 8 & - & 40 & 20.70 & \pm & 8.49 & & $\mathrm{P} 2$ & 0.001 & P7 & 0.004 \\
\hline & Group C & 24 & - & 286 & 98.12 & \pm & 68.21 & & P3 & 0.001 & P8 & 0.022 \\
\hline & Group D & 12 & - & 227 & 69.39 & \pm & 48.49 & & $\mathrm{P} 4$ & 0.012 & P9 & 0.009 \\
\hline & Group E & 16 & - & 121 & 59.33 & \pm & 30.39 & & P5 & 0.001 & P10 & 0.464 \\
\hline \multirow{5}{*}{ AST } & Group A & 12 & - & 41 & 28.07 & \pm & 9.86 & \multirow{5}{*}{0.001} & $\mathrm{P} 1$ & 0.756 & P6 & 0.001 \\
\hline & Group B & 6 & - & 40 & 23.82 & \pm & 8.83 & & $\mathrm{P} 2$ & 0.001 & P7 & 0.001 \\
\hline & Group C & 28 & - & 210 & 78.76 & \pm & 51.97 & & P3 & 0.001 & P8 & 0.821 \\
\hline & Group D & 8.5 & - & 337 & 81.93 & \pm & 69.19 & & $\mathrm{P} 4$ & 0.005 & P9 & 0.923 \\
\hline & Group E & 22 & - & 216 & 77.17 & \pm & 51.51 & & P5 & 0.001 & P10 & 0.760 \\
\hline \multirow{5}{*}{$\begin{array}{c}\text { Total } \\
\text { Bilirubin }\end{array}$} & Group A & 0.3 & - & 1.1 & 0.63 & \pm & 0.27 & \multirow{5}{*}{0.001} & $\mathrm{P} 1$ & 0.957 & P6 & 0.651 \\
\hline & Group B & 0.2 & - & 1.3 & 0.61 & \pm & 0.30 & & $\mathrm{P} 2$ & 0.665 & P7 & 0.001 \\
\hline & Group C & 0.3 & - & 1.2 & 0.75 & \pm & 0.29 & & $\mathrm{P} 3$ & 0.749 & P8 & 0.882 \\
\hline & Group D & 0.2 & - & 1.3 & 0.71 & \pm & 0.31 & & $\mathrm{P} 4$ & 0.001 & P9 & 0.001 \\
\hline & Group E & 0.3 & - & 8.2 & 1.83 & \pm & 2.11 & & P5 & 0.586 & P10 & 0.001 \\
\hline \multirow{5}{*}{$\begin{array}{c}\text { Direct } \\
\text { Bilirubin }\end{array}$} & Group A & 0.07 & - & 0.5 & 0.19 & \pm & 0.13 & \multirow{5}{*}{0.001} & $\mathrm{P} 1$ & 0.910 & P6 & 0.905 \\
\hline & Group B & 0.1 & - & 0.9 & 0.22 & \pm & 0.21 & & $\mathrm{P} 2$ & 0.807 & P7 & 0.001 \\
\hline & Group C & 0.1 & - & 0.8 & 0.26 & \pm & 0.19 & & P3 & 0.838 & P8 & 0.953 \\
\hline & Group D & 0.09 & - & 0.8 & 0.24 & \pm & 0.18 & & $\mathrm{P} 4$ & 0.001 & P9 & 0.001 \\
\hline & Group E & 0.1 & - & 7.85 & 1.30 & \pm & 2.15 & & P5 & 0.864 & P10 & 0.001 \\
\hline \multirow{5}{*}{$\begin{array}{c}\text { Serum } \\
\text { Albumin }\end{array}$} & Group A & 3.5 & - & 5.1 & 4.18 & \pm & 0.47 & \multirow{5}{*}{0.001} & $\mathrm{P} 1$ & 0.014 & P6 & 0.017 \\
\hline & Group B & 2.6 & - & 4.6 & 3.72 & \pm & 0.48 & & $\mathrm{P} 2$ & 0.024 & P7 & 0.001 \\
\hline & Group C & 2.5 & - & 4.79 & 3.70 & \pm & 0.62 & & P3 & 0.001 & P8 & 0.051 \\
\hline & Group D & 1.9 & - & 4.5 & 3.32 & \pm & 0.68 & & $\mathrm{P} 4$ & 0.001 & P9 & 0.001 \\
\hline & Group E & 0.4 & - & 3.4 & 2.47 & \pm & 0.78 & & $\mathrm{P} 1$ & 0.014 & P6 & 0.017 \\
\hline
\end{tabular}

P1: Group A \& Group B. P2: Group A \& Group C. P3: Group A \& Group D.

P4: Group A \& Group E. P5: Group B \& Group C. P6: Group B \& Group D.

P7: Group B \& Group E. P8: Group C \& Group D. P9: Group C \& Group E.

P10: Group D \& Group E. SD: Standard deviation. 
The mean values of PT and INR in group E were $17.01 \pm 5.16$ and $1.44 \pm 0.45$ respectively. There were significant increases of PT and INR in group E when compared with group $\mathrm{A}$ with $\mathrm{P}$ value
$<0.05$. Kruskal-Wallis test showed significant difference among all groups regarding the PT and INR with $\mathrm{P}$ value $<0.05$ in both PT and INR (Table 5).

Table (5): Means and standard deviations of some variables of bleeding profile and comparison between all groups

\begin{tabular}{|c|c|c|c|c|c|c|c|c|c|c|c|c|}
\hline \multicolumn{2}{|c|}{$\begin{array}{l}\text { Parameters } \\
\text { Variables }\end{array}$} & \multicolumn{3}{|c|}{ Range } & \multirow{2}{*}{$\begin{array}{c}\text { Mean } \\
13.38\end{array}$} & \multirow{2}{*}{$\begin{array}{l} \pm \\
\pm\end{array}$} & \multirow{2}{*}{$\begin{array}{l}\text { S. D } \\
1.44\end{array}$} & $\begin{array}{c}\text { p. } \\
\text { value }\end{array}$ & \multicolumn{4}{|c|}{ Post Hock test (LSD) } \\
\hline \multirow{5}{*}{ PT } & Group A & 11.9 & - & 18.2 & & & & \multirow{5}{*}{0.013} & $\mathrm{P} 1$ & 0.353 & P6 & 0.432 \\
\hline & Group B & 10.6 & - & 32.4 & 14.38 & \pm & 3.81 & & P2 & 0.555 & P7 & 0.027 \\
\hline & Group C & 11.7 & - & 19.2 & 14.11 & \pm & 2.21 & & P3 & 0.131 & P8 & 0.358 \\
\hline & Group D & 11.7 & - & 28.7 & 15.13 & \pm & 3.51 & & $\mathrm{P} 4$ & 0.008 & P9 & 0.028 \\
\hline & Group E & 13 & - & 31.8 & 17.01 & \pm & 5.16 & & P5 & 0.787 & $\mathrm{P} 10$ & 0.129 \\
\hline \multirow{5}{*}{ INR } & Group A & 0.9 & - & 1.9 & 1.16 & \pm & 0.23 & \multirow{5}{*}{0.042} & $\mathrm{P} 1$ & 0.771 & P6 & 0.308 \\
\hline & Group B & 1 & - & 2.73 & 1.19 & \pm & 0.33 & & $\mathrm{P} 2$ & 0.872 & $\mathrm{P} 7$ & 0.022 \\
\hline & Group C & 0.8 & - & 1.8 & 1.18 & \pm & 0.25 & & $\mathrm{P} 3$ & 0.269 & P8 & 0.332 \\
\hline & Group D & 0.91 & - & 2.27 & 1.28 & \pm & 0.28 & & $\mathrm{P} 4$ & 0.026 & P9 & 0.032 \\
\hline & Group E & 1 & - & 2.5 & 1.44 & \pm & 0.45 & & P5 & 0.911 & $\mathrm{P} 10$ & 0.156 \\
\hline
\end{tabular}

P1: Group A \& Group B. P2: Group A \& Group C. P3: Group A \& Group D.

P4: Group A \& Group E. P5: Group B \& Group C. P6: Group B \& Group D.

P7: Group B \& Group E. P8: Group C \& Group D. P9: Group C \& Group E.

P10: Group D \& Group E. SD: Standard deviation.

Mann Whitney U test was conducted to test the significant difference between recovered and died for all cases regarding some variables of liver functions tests. The results revealed no significant differences between recovered and died mean values of the ALT for all cases P- value >0.05. However, there was a significant difference between recovered and died mean values of the AST, total bilirubin, direct bilirubin and serum albumin for all cases with $\mathrm{P}$-value $<0.05$ for all cases (Table 6).

Table (6): Comparison of some variables of liver functions tests between recovered and died for all cases among COVID 19 patients

\begin{tabular}{|c|c|c|c|c|c|c|c|c|}
\hline \multicolumn{2}{|c|}{$\begin{array}{ll} & \text { Parameters } \\
\text { Variables } & \end{array}$} & \multicolumn{3}{|c|}{ Range } & \multirow{2}{*}{$\begin{array}{c}\text { Mean } \\
46.52\end{array}$} & \multirow[t]{2}{*}{ \pm} & \multirow{2}{*}{$\frac{\text { S. D }}{45.97}$} & \multirow{3}{*}{$\begin{array}{c}\text { p. value } \\
0.053\end{array}$} \\
\hline \multirow{2}{*}{ ALT } & Recovered & 6 & - & 286 & & & & \\
\hline & Died & 15 & - & 227 & 73.75 & \pm & 59.02 & \\
\hline \multirow{2}{*}{ AST } & Recovered & 6 & - & 210 & 46.46 & \pm & 37.26 & \multirow{2}{*}{0.017} \\
\hline & Died & 17 & - & 337 & 105.67 & \pm & 94.74 & \\
\hline \multirow{2}{*}{$\begin{array}{c}\text { Total } \\
\text { Bilirubin }\end{array}$} & Recovered & 0.2 & - & 2.2 & 0.69 & \pm & 0.36 & \multirow{2}{*}{0.004} \\
\hline & Died & 0.5 & - & 8.2 & 1.66 & \pm & 2.13 & \\
\hline \multirow{2}{*}{$\begin{array}{c}\text { Direct } \\
\text { Bilirubin }\end{array}$} & Recovered & 0.07 & - & 1.4 & 0.25 & \pm & 0.23 & \multirow{2}{*}{0.024} \\
\hline & Died & 0.1 & - & 7.85 & 1.14 & \pm & 2.19 & \\
\hline \multirow{2}{*}{$\begin{array}{l}\text { Serum } \\
\text { Albumin }\end{array}$} & Recovered & 0.4 & - & 5.1 & 3.60 & \pm & 0.74 & \multirow{2}{*}{0.032} \\
\hline & Died & 1.8 & - & 4.1 & 3.11 & \pm & 0.75 & \\
\hline
\end{tabular}


Pearson correlation coefficient was conducted to determine the relationship between ALT and AST. Results revealed a positive correlation between ALT and
AST for groups C, D and E ( $\mathrm{r}=0.805$, 0.676 and 0.813 respectively and for all cases $r=0.766$ ) (Table 7).

Table (7): Correlation between ALT and AST among COVID-19 patients

\begin{tabular}{|c|c|c|}
\hline ALT & $\boldsymbol{r}$ & $\boldsymbol{p}$ \\
\hline Group A & 0.161 & 0.568 \\
\hline Group B & 0.253 & 0.155 \\
\hline Group C & 0.805 & 0.001 \\
\hline Group D & 0.676 & 0.001 \\
\hline Group E & 0.813 & 0.001 \\
\hline Total & 0.766 & 0.001 \\
\hline
\end{tabular}

r: Pearson correlation.

p: probability value.

The results showed that a significant difference ( $p$-value <0.05) were found between recovered and died cases for each group with higher mortality rate at group D (7 cases with a percent of $30.4 \%)$ then at group E ( 3 cases with a percent of $25 \%$ ) and there was 88 cases $(88 \%)$ recovered and 12 cases $(12 \%)$ died in all studied COVID-19 patients (Table 8).

Table (8): Comparison of mortality rate between recovered and died cases for all cases and each group among COVID-19 patients

\begin{tabular}{|c|c|c|c|c|c|c|c|}
\hline \multicolumn{2}{|c|}{$\begin{array}{l}\text { Groups } \\
\text { Mortality rate }\end{array}$} & $\begin{array}{c}\text { Group A } \\
(\mathbf{n}=\mathbf{1 5})\end{array}$ & $\begin{array}{c}\text { Group B } \\
(\mathbf{n}=\mathbf{3 3})\end{array}$ & $\begin{array}{c}\text { Group C } \\
(n=17)\end{array}$ & $\begin{array}{c}\text { Group D } \\
(n=23)\end{array}$ & $\begin{array}{c}\text { Group E } \\
(n=12)\end{array}$ & P-value \\
\hline \multirow{2}{*}{$\begin{array}{c}\text { Recovered } \\
(\mathbf{n}=\mathbf{8 8})\end{array}$} & $\mathbf{N}$ & 15 & 32 & 16 & 16 & 9 & \multirow{4}{*}{0.006} \\
\hline & $\%$ & $100.0 \%$ & $97.0 \%$ & $4.1 \%$ & $9.6 \%$ & $75.0 \%$ & \\
\hline \multirow{2}{*}{$\begin{array}{c}\text { Died } \\
(n=12)\end{array}$} & $\mathbf{N}$ & 0 & 1 & 1 & 7 & 3 & \\
\hline & $\%$ & $.0 \%$ & $3.0 \%$ & $5.9 \%$ & $30.4 \%$ & $25.0 \%$ & \\
\hline
\end{tabular}

$\mathrm{N}$ : number of cases. 
There were significant differences ( $\mathrm{p}$ value $<0.05$ ) between recovered patients and died patients as regard to CT chest. There were radiological findings suggesting that COVID-19 in CT chest of all including cases with higher mortality rate at group $\mathrm{D}$ ( 7 cases with a percent of $30.4 \%$ and 5 cases of them with score 5 with a percent of $21.7 \%$ ), then at group $\mathrm{E}$
( 3 cases with a percent of $25 \%$ and 2 cases of them with score 5 with a percentage of $16.6 \%)$ and there was 88 cases $(88 \%)$ recovered and 12 cases (12\%) died in all studied COVID-9 patients. The results revealed that the higher mortality rate at group D and $\mathrm{E}$ between patients having chest CT scoring 4 and 5 (Table 9).

Table (9): Comparison CT findings between recovered and died cases for all COVID-19 patients.

\begin{tabular}{|c|c|c|c|c|c|c|c|c|}
\hline CT findings & & ore & $\begin{array}{c}\text { Score } \\
1\end{array}$ & $\begin{array}{c}\text { Score } \\
2\end{array}$ & $\begin{array}{c}\text { Score } \\
3\end{array}$ & $\begin{array}{c}\text { Score } \\
4\end{array}$ & $\begin{array}{c}\text { Score } \\
5\end{array}$ & $\begin{array}{c}\mathbf{P -} \\
\text { value }\end{array}$ \\
\hline \multirow{4}{*}{$\begin{array}{c}\text { Group A } \\
(n=15)\end{array}$} & \multirow{2}{*}{$\begin{array}{c}\text { Recovered } \\
(n=15)\end{array}$} & $\mathbf{N}$ & 6 & 3 & 3 & 2 & 1 & \multirow[t]{4}{*}{ 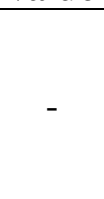 } \\
\hline & & $\%$ & $40.0 \%$ & $20.0 \%$ & $20.0 \%$ & $13.3 \%$ & $6.6 \%$ & \\
\hline & \multirow{2}{*}{$\begin{array}{c}\text { Died } \\
(\mathbf{n}=0)\end{array}$} & $\mathbf{N}$ & 0 & 0 & 0 & 0 & 0 & \\
\hline & & $\%$ & $.0 \%$ & $.0 \%$ & $.0 \%$ & $.0 \%$ & $.0 \%$ & \\
\hline \multirow{4}{*}{$\begin{array}{c}\text { Group B } \\
(\mathbf{n}=\mathbf{3 3})\end{array}$} & \multirow{2}{*}{$\begin{array}{c}\text { Recovered } \\
(\mathbf{n}=\mathbf{3 2})\end{array}$} & $\mathbf{N}$ & 2 & 3 & 8 & 9 & 10 & \multirow{4}{*}{0.668} \\
\hline & & $\%$ & $6.0 \%$ & $9.0 \%$ & $24.2 \%$ & $27.2 \%$ & $30.3 \%$ & \\
\hline & \multirow{2}{*}{$\begin{array}{l}\text { Died } \\
(n=1)\end{array}$} & $\mathbf{N}$ & 0 & 0 & 0 & 1 & 0 & \\
\hline & & $\%$ & $.0 \%$ & $.0 \%$ & $.0 \%$ & $3.0 \%$ & $.0 \%$ & \\
\hline \multirow{4}{*}{$\begin{array}{c}\text { Group C } \\
(\mathbf{n}=17)\end{array}$} & \multirow{2}{*}{$\begin{array}{c}\text { Recovered } \\
(\mathrm{n}=16)\end{array}$} & $\mathbf{N}$ & 3 & 3 & 4 & 3 & 3 & \multirow{4}{*}{0.485} \\
\hline & & $\%$ & $17.6 \%$ & $17.6 \%$ & $23.5 \%$ & $17.6 \%$ & $17.6 \%$ & \\
\hline & \multirow{2}{*}{$\begin{array}{l}\text { Died } \\
(n=1)\end{array}$} & $\mathbf{N}$ & 0 & 0 & 0 & 1 & 0 & \\
\hline & & $\%$ & $.0 \%$ & $.0 \%$ & $.0 \%$ & $5.8 \%$ & $.0 \%$ & \\
\hline \multirow{4}{*}{$\begin{array}{c}\text { Group D } \\
(\mathbf{n}=\mathbf{2 3})\end{array}$} & \multirow{2}{*}{$\begin{array}{c}\text { Recovered } \\
(n=16)\end{array}$} & $\mathbf{N}$ & 1 & 6 & 2 & 4 & 3 & \multirow{4}{*}{0.125} \\
\hline & & $\%$ & $2.3 \%$ & $26.0 \%$ & $8.6 \%$ & $17.3 \%$ & $13.0 \%$ & \\
\hline & \multirow{2}{*}{$\begin{array}{l}\text { Died } \\
(n=7)\end{array}$} & $\mathbf{N}$ & 0 & 0 & 1 & 1 & 5 & \\
\hline & & $\%$ & $.0 \%$ & $.0 \%$ & $2.3 \%$ & $2.3 \%$ & $21.7 \%$ & \\
\hline \multirow{4}{*}{$\begin{array}{c}\text { Group } E \\
(n=12)\end{array}$} & \multirow{2}{*}{$\begin{array}{c}\text { Recovered } \\
(\mathbf{n}=9)\end{array}$} & $\mathbf{N}$ & 2 & 1 & 2 & 2 & 2 & \multirow{4}{*}{0.647} \\
\hline & & $\%$ & $16.6 \%$ & $8.3 \%$ & $16.6 \%$ & $16.6 \%$ & $16.6 \%$ & \\
\hline & \multirow{2}{*}{$\begin{array}{c}\text { Died } \\
(\mathbf{n}=\mathbf{3})\end{array}$} & $\mathbf{N}$ & 0 & 0 & 0 & 1 & 2 & \\
\hline & & $\%$ & $.0 \%$ & $.0 \%$ & $.0 \%$ & $8.3 \%$ & $16.6 \%$ & \\
\hline \multirow{4}{*}{$\begin{array}{l}\text { All Cases } \\
(\mathbf{n}=\mathbf{1 0 0})\end{array}$} & \multirow{2}{*}{$\begin{array}{c}\text { Recovered } \\
\quad(n=88)\end{array}$} & $\mathbf{N}$ & 14 & 16 & 19 & 20 & 19 & \multirow{4}{*}{0.027} \\
\hline & & $\%$ & $14.0 \%$ & $16.0 \%$ & $19.0 \%$ & $20.0 \%$ & $19.0 \%$ & \\
\hline & \multirow{2}{*}{$\begin{array}{c}\text { Died } \\
(n=12)\end{array}$} & $\mathbf{N}$ & 0 & 0 & 1 & 4 & 7 & \\
\hline & & $\%$ & $.0 \%$ & $.0 \%$ & $1.0 \%$ & $4.0 \%$ & $7.0 \%$ & \\
\hline
\end{tabular}

$\mathrm{N}$ : number of cases.

\section{DISCUSSION}

In the present study, it was found that the mean of the neutrophil /lymphocyte ratio reaching to a maximum mean value at group $\mathrm{E}$ and the lymphocyte /monocyte ratio reaching to a minimum mean value of $2.13 \pm 1.80$ at group E. Our results were in agreement with Chan and Rout (2020) who stated that patients with severe COVID-19 disease had higher 
NLR value compared to non-severe disease and level of NLR correlated with COVID-19 disease severity. Also in our study, it was found that the lymphocyte /monocyte ratio reaching to a minimum mean value at group E. Our results agreed with Liu et al. (2020-a) who reported that $\mathrm{T}$ helper cells induce the production of cytokines such as interleukin-17 through the nuclear factor kappa light-chainenhancer of activated $\mathrm{B}$ cells (NF-kB) signaling pathway, leading to increased aggregation of monocytes. SARS-CoV-2 infects circulating immune cells and increases apoptosis of lymphocytes, leading to lymphopenia. A lower ratio of circulating lymphocytes to monocytes (LMR) predicts severe and extremely severe COVID-19 as the clearance of the virus is delayed due to lymphopenia and also a decrease in CD4+ T cells. Either a rise or fall in lymphocyte levels is an extremely crucial prognostic indicator of mortality in COVID-19.

NLR elevation was found to be associated with poor outcomes in myocardial infarction, coronary artery disease, atherosclerosis, chronic obstructive pulmonary disease (COPD) and high nuclear grade renal cell carcinoma (RCC) (Hu et al., 2015 and Ye et al., 2019). LMR level has been correlated with several malignancies that might have complex interplay between immune system and oncogenesis. Their prognostic effectiveness has been investigated in hematological malignancies and solid tumors such as colon, bladder, and lung cancers (Ye et al., 2019). Moreover, a study from Italy proved that COVID-19 patients had higher monocyte absolute count values compared to patients with flu, suggesting a potential prognostic role of monocyte macrophages activation ( $\mathrm{Gu}$ et al., 2016 and Curtoloet al., 2020).

Patients with sustained elevated NLR had worse results in almost all measured outcomes. An inflammatory response activates the local innate immunity of the body to provide protection against ingress of microorganisms. However, abnormalities can occur in host defense systems in response to infection. A dysregulated or unbalanced inflammatory response escalates and releases an excess of pro-inflammatory mediators such as IL1, IL-6, IL-8, and TNF a, which can result in systemic inflammatory response syndrome (Tatum et al., 2020). Progression of this cascade and hyperinflammation worsens the initial burden. High levels of neutrophil infiltration and systemic circulation result in increased systemic arginase activity, which results in depleted systemic arginine (Caldwell et al., 2018). Arginine is the sole substrate for nitric oxide (NO) production, which has known antiviral activity against RNA viruses such as SARS-CoV-2 (Luiking et al., 2012 and Caldwell et al., 2018).

In the present work, our resulted revealed that there was a significant increase of ALT and AST mean values in groups C, D and E when compared with group A. Also, our results showed that there was a significant increase of total bilirubin, direct bilirubin mean values in group E when compared with group A. There were significant decrease of serum albumin mean value in groups $\mathrm{C}, \mathrm{D}$ and $\mathrm{E}$ when compared with group A with a minimum mean value in group $\mathrm{E}$, there was a significant increase of PT and INR 
mean values in groups $\mathrm{E}$ when compared with group A. These results agreed with Ghoda \& Ghoda (2020) and Kullar et al. (2020) who found that regardless of existing CLD, COVID-19 was associated with mild to moderate liver failure, reflected mainly by hypertransaminasemia, elevation of gamma-glutamyl transferase (GGT) and alkaline phosphatase (ALP) levels (less frequently), hypoproteinemia and prolonged prothrombin time.

In our results, no significant difference between recovered and died mean values of the ALT for all cases. However, there were significant difference between recovered and died mean values of the AST, total bilirubin, direct bilirubin and serum albumin for all cases, and were associated with severity and mortality among COVID-19 patients, but there was a positive correlation between ALT and AST for groups C, D and E and for all cases. Our findings were in agreement with Alqahtani et al. (2020), Chen et al. (2020) and Ghweil et al. (2020).

Some reports have even proved the presence of a correlation between abnormal liver tests and coagulation dysfunction in SARS-CoV-2 pneumonia, highlighting the significant role of the liver in this disease (Chen et al., 2020). However, the data are too scanty to differentiate an exact background of hypertransaminasemia in COVID-19 patients with a pre-existing chronic liver failure or a certain hepatotoxic impact of SARS-CoV-2 infection. The catastrophic emergence of COVID-19 has led to large volumes of research from epicentres of infection, with some focusing on COVID19-related liver impairment. In this regard,
Wang et al. (2020) found that very minimal elevations in alanine (ALT) and aspartate aminotransferase (AST; ALT $>$ AST) to disease severity and demonstrate 'specific' COVID-19-related cytopathic changes and virus-like particles on post-mortem liver histopathology. Furthermore, they found that severe acute respiratory syndrome coronavirus 2 (SARS-Cov-2) caused massive apoptosis and binucleation of hepatocytes, resulting in liver enzyme abnormality and synthetic liver dysfunction, the latter in the form of hypoalbuminemia. Their painstaking work is commendable, but their assessment of clinical and investigational events may not reflect the reality.

Philips et al. (2020) reported that the degree of elevation in AST and ALT can only be considered an 'altered' liver test, not akin to acute hepatic injury. In hepatic impairment, there must be very clear evidence for metabolic (hypoglycaemia, hyperammonemia), secretory (hyperbilirubinemia) and synthetic (hypoalbuminemia, raised prothrombin time) dysfunction. Except for a mild rise in ALT and hypoalbuminemia, significant liver dysfunction is elusive in the current study. Importantly, hypoalbuminemia, in the absence of other significant liver test abnormalities, virtually rules out the hepatic origin of this abnormality.

Acute liver injury is best identified by international normalized ratio $>2.0$ (Koch et al., 2017). The liver biopsy findings of hepatocyte apoptosis, binuclear or occasional multinuclear syncytial hepatocytes, in the absence of viral inclusions and presence of moderate steatosis, with mild focal lobular or portal inflammation are non-specific findings 
that may not be related to viral cytopathy. These findings can undoubtfully be seen in sepsis and multi-organ dysfunction associated with critical illness (moderate to severe apoptosis, steatosis, lobular and portal inflammation), aging, drug-induced liver injury and fatty liver disease (binucleation or polyploidy- a feature of liver cell renewal and not injury) (Hsu and Duncan, 2015).

The COVID-19 related liver injury is defined as any liver damage occurring during disease progression and treatment of COVID-19 in patients with or without pre-existing liver disease (Sun et al., 2020). The impact of COVID-19 virus infection on the liver is still unclear. Several studies have shown that COVID19 virus infection causes abnormality in liver function proven by elevation of serum liver biochemistries such as alanine aminotransferase (ALT), aspartate aminotransferase (AST), and total bilirubin. The incidence of elevated ALT and AST are ranged from $2.5 \%-50 \%$ to $2.5 \%-61.1 \%$, respectively (Ridruejo and Soza, 2020). Low of albumin level is also a marker of severe infection and poor prognosis (Giuliano, 2020).

In the present work, our results showed that a significant difference were found between recovered and died cases for each group as regard to mortality rate with higher mortality rate was found at group D $(30.4 \%)$, then at group E $(25 \%)$ and there was $88 \%$ recovered and $12 \%$ died in all studied COVID-9 patients. Our results were in a line with Liu et al. (2020) and Gholizadehet al. (2020) who reported that the accumulated data suggest that more than one-third of patients hospitalized due to SARS-CoV-2 infection might have impaired liver function. An increase in aspartate transaminase (AST) and alanine transaminase (ALT) activity, especially in men, results in a severe course of COVID19. In general, a higher level of ALT, thrombocytopenia and hypoalbuminemia are indices of increased mortality in COVID-19 patients. Moreover, hypoalbuminemia is recognized as an independent marker of severe SARSCoV-2 infection, poor prognosis and higher mortality. Also our results were in concomitant with Sulaiman et al. (2020) who stated that the mortality rate of COVID-19 virus infection in patients with pre-existing chronic liver disease and cirrhosis found that patients with chronic liver disease and cirrhosis had clinical factors associated with poor outcomes from COVID-19 virus infection.

Recent investigations on complications of COVID-19 have revealed that the occurrence of liver injury ranged from $14.8 \%$ to $53 \%$. Also, it is accompanied mainly by abnormal ALT/AST levels followed by slightly elevated bilirubin levels (Xu et al., 2020). The proportion of liver injury in death cases and severe COVID-19 patients was significantly higher than that in mild patients (Huang et al., 2020).

Concerning CT chest, our results showed that the findings were bilateral peripheral ground glass opacity among all cases who affected by COVID-19 infection, and it was found that there was a significant difference between recovered patients and died patients as regard to $\mathrm{CT}$ chest. There were radiological findings suggested COVID-19 in CT chest of all including cases with higher mortality rate was found at group D $30.4 \%$ out of them 
$21.7 \%$ with $\mathrm{CT}$ chest scoring 5 then at group E $25 \%$ out of them $16.6 \%$ with CT chest scoring 5 and there was $88 \%$ recovered and $12 \%$ died in all studied COVID-9 patients. Our results revealed that the higher mortality rate was found in Group D and E between patients having CT chest scoring 4 and 5. The same findings were reported by multiple studies (Ghweil et al., 2020; Majidi \& Niksolate, 2020; Song et al., 2020 and Wong et al., 2020). So, CT chest findings were predictors in the severity and mortality of COVID-19 infection.

\section{CONCLUSION}

Impaired liver diagnostic test results constitute common findings in COVID-19 patients. Deterioration of liver function worsens the prognosis, increases the risk of severe SARS-CoV-2 infection and prolongs the duration of hospitalization. Abnormal liver function test results may be predictors of COVID-19 severity and mortality rate.

\section{REFERENCES}

1. Alqahtani JS, Oyelade T, Aldhahir AM, Alghamdi S, Almehmadi M, Alqahtani A, Quaderi S, Mandal S and Hurst J (2020): Prevalence, severity and mortality associated with COPD and Smoking in patients with COVID-19: A rapid systematic review and meta- analysis. PLoS One, 15(5): e0233147.

2. Caldwell RW, Rodriguez PC, Toque HA, Narayanan SP and Caldwell RB (2018): Arginase: a multifaceted enzyme important in health and disease. Physiol Rev., 98(2): 641665.

3. Chan AS and Rout A (2020): Use of Neutrophil-to-Lymphocyte and Platelet- to Lymphocyte Ratios in COVID-19. J. Clin Med Res., 12(7):448-453.

4. Chen S, Liu H, Li T, Huang R, Gui R and Zhang $J$ (2020): Correlation analysis of coagulation dysfunction and liver damage in patients with novel coronavirus pneumonia: a single-center, retrospective, observational study. Ups J Med Sci., 125(4): 293-296.

5. Curtolo A, Oliva A, Volpicelli L, Ceccarelli G, D'Ettorre G, Borrazzo C, Mastroianni CM and Venditti M (2020): Monocyte absolute count as a preliminary tool to distinguish between SARS-CoV-2 and influenza $\mathrm{A} / \mathrm{B}$ infections in patients requiring hospitalization. Infez Med., 28(4): 534-538.

6. Ghoda A and Ghoda M (2020): Liver Injury in COVID-19 Infection: A Systematic Review. Cureus., 12(7): e9487.

7. Gholizadeh P, Safari R, Marofi P, Zeinalzadeh E, Pagliano P, Ganbarov K, Esposito S, Khodadadi E, Yousefi $M$ and SamadiKafil H (2020): Alteration of Liver Biomarkers in Patients with SARS-CoV-2 (COVID-19). J Inflamm Res., 13: 285-292.

8. Ghweil AA, Hassan MH, Khodeary A, Mohamed AO, Mohamed HM, Abdelazez AA, Osman HA and Bazeed SES (2020): Characteristics, Outcomes and Indicators of Severity for COVID-19 Among Sample of ESNA Quarantine Hospital's Patients, Egypt: A Retrospective Study. Infection and Drug Resistance, 13: 2375-2383.

9. Giuliano R. (2020): Hypoalbuminemia: an underestimated, viral characteristic of hospitalized COVID-19 positice patients?. Hepatoma Res., 6(28): 1-10.

10. Gu, L., Li, H., Chen, L., Ma, X., Li, X., Gao, Y., Zhang, Y,. Xie, Y. and Zhang, X. (2016): Prognostic role of lymphocyte to monocyte ratio for patients with cancer: evidence from a systematic review and metaanalysis. Oncotarget., 7(22): 31926-31942.

11. Hsu SH. and Duncan AW. (2015): Pathological polyploidy in liver disease. Hepatology, 62(3): 968-970.

12. Hu K, Lou L, Ye J and Zhang $S$ (2015): Prognostic role of the neutrophil-lymphocyte ratio in renal cell carcinoma: a meta-analysis. BMJ Open, 5 (4): e006404.

13. Huang, C., Wang, Y., Li, X., Ren, L., Zhao, J., Hu, Y., Zhang, L., Fan, G., Xu, J., Gu, X., Cheng, Z., Yu, T., Xia, J., Wei, Y., Wu, 
W., Xie, X., Yin, W., Li, H., Liu, M., Xiao, Y., Gao, H., Guo, L., Xie, J., Wang, G., Jiang, R., Gao, Z., Jin, Q., Wang, J. and Cao, B. (2020): Clinical features of patients infected with 2019 novel coronavirus in Wuhan, China. The Lancet, 395(10223): 497 506.

14. Koch, D.G., Speiser, J.L., Durkalski, V., Fontana, R.J., Davern, T., McGuire, B., Stravitz, R.T., Larson, A.M. , Liou, I., Fix, O., Schilsky, M.L., McCashland, T., Hay, J.E., Murray, N., Shaikh, O.S., Ganger, D., Zaman, A., Han, S.B., Chung, R.T., Brown, R.S., Munoz, Jr., Reddy, K.R., Rossaro, L., Satyanarayana, R., Hanje, A.J., Olson, J., Subramanian, R.M., Karvellas, C., Hameed, B., Sherker, A.H., Lee, W.M. and Reuben, A. (2017): The Natural History of Severe Acute Liver Injury. The American Journal of Gastroenterology, 112(9): 1389-1396.

15. Kullar R, Patel AP and Saab S (2020): Hepatic Injury in Patients with COVID-19. J Clin Gastroenterol., 54(10): 841-849.

16. Liu G, Zhang S, Hu H, Liu TT and Huang J (2020-a): The role of neutrophillymphocyte ratio and lymphocyte-monocyte ratio in the prognosis of type 2 diabetics with COVID-19. Scott Med J., 65(4): 154-160.

17. Liu W, Tao ZW, Wang L, Yuan ML, Liu K, Zhou L, Wei S, Deng Y, Liu J, Liu HG, Yang $M$ and Hu Y (2020-b): Analysis of factors associated with disease outcomes in hospitalized patients with 2019 novel coronavirus disease. Chin Med J (Engl)., 133: 1032-1038.

18. Liu Y, Gayle AA, Wilder-Smith A and Rocklöv J (2020-c): The reproductive number of COVID-19 is higher compared to SARS coronavirus. J Travel Med., 27(2): 1-4.

19. Luiking YC, Ten Have GA, Wolfe RR and Deutz NE (2012): Arginine de novo and nitric oxide production in disease states. Am J Physiol Endocrin Metab., 303(10): E1177E1189.

20. Majidi $H$ and Niksolat F (2020): Chest CT in patients suspected of COVID-19 infection: A reliable alternative for RT- PCR. American
Journal of Emergency Medicine, 38(12): 2730-2732.

21. Pan Y, Zhang D, Yang P, Poon LLM and Wang Q (2020): Viral load of SARS-CoV-2 in clinical samples. Lancet Infect Dis., 20(4): 411-412.

22. Philips CA, Ahamed $\mathbf{R}$ and Augustine $\mathbf{P}$ (2020): SARS-CoV-2 related liver impairment-perception may not be the reality. Journal of Hepatology, 73: 967-998.

23. Qin $\mathrm{C}$, Zhou $\mathrm{L}$, Hu $\mathrm{Z}$, Zhang $\mathrm{S}$, Yang $\mathrm{S}$, Tao Y, Xie C, Ma K, Shang K, Wang W and Tian DS (2020): Dysregulation of Immune Response in Patients With Coronavirus 2019 (COVID-19) in Wuhan, China. Clin Infect Dis., 71(15): 762-768.

24. Ridruejo E and Soza A (2020): The liver in times of COVID-19: What hepatologists should know. Ann Hepatol., 19(4): 353-358.

25. Salehi S, Abedi A, Balakrishnan $S$ and Gholamrezanezhad A (2020): Coronavirus disease 2019 (COVID-19): A systematic review of imaging findings in 919 patients. Am J Roentgenol., 215(1): 87-93.

26. Soeters PB, Wolfe RR and Shenkin A (2019): Hypoalbuminemia: Pathogenesis and Clinical Significance. JPEN J Parenter Enteral Nutr., 43(2):181-193.

27. Song F, Shi N, Shan F, Zhang Z, Shen J, Lu H, Ling Y, Jiang Y and Shi Y (2020): Emerging coronavirus 2019- $\mathrm{nCoV}$ pneumonia. Radiology, 295(1): 210-217.

28. Sulaiman AS, Kurniawan J, Chyntia OM, Saut HH, Kemal F, Cosmas Rinaldi A, Hasan I, Gani RA and Baiq Kirana DN (2020): COVID-19 Related to Liver Impairment and Its Impact on Chronic Liver Disease. The Indonesian Journal of Gastroenterology, Hepatology and Digestive Endoscopy, 21(3): 220-223.

29. Sun J, Aghemo A, Forner A and Valenti L (2020): COVID-19 and liver disease. Liver International, 40: 1278.

30. Tatum D, Taghavi S, Houghton A, Stover J, Toraih E and Duchesne J (2020): Neutrophil-to-lymphocyte ratio and outcomes 
in Louisiana Covid-19 patients. Shock, 54(5): 652-658.

31. Wang Y, Liu S, Liu H, Li W, Lin F, Jiang L, Li X, Xu P, Zhang L, Zhao L, Cao Y, Kang J, Yang J, Li L, Liu X, Li Y, Nie R, Mu J, Lu F, Zhao S, Lu J and Zhao J (2020): SARS-CoV-2 infection of the liver directly contributes to hepatic impairment in patients with COVID-19. J Hepatol., 73(4): 807-816.

32. Wong HYF, Lam HYS, Fong AHT, Leung ST, Chin TWY, Lo CSY, Lui MMS, Lee JHY, Chiu KWH, Chung TWH, Lee EYP, Wan EYF, Hung IFN, Lam TPW, Kuo MD and Ng MY (2020): Frequency and distribution of chest radiographic findings in COVID-19 positive patients. Radiology, 296(2): E72-E78.
33. Xu L, Liu J, Lu M, Yang $D$ and Zheng $X$ (2020): Liver injury during highly pathogenic human coronavirus infections. Liver Int., 40 (5): 998-1004.

34. Ye Z, Ai X, Liao Z, You $C$ and Cheng $Y$ (2019): The prognostic values of neutrophil to lymphocyte ratio for outcomes in chronic obstructive pulmonary disease. Medicine (Baltimore), 98(28): e16371.

35. Zhang Y, Zheng L, Liu L, Zhao M, Xiao J and Zhao $Q$ (2020): Liver impairment in COVID-19 patients: a retrospective analysis of 115 cases from a single center in Wuhan city, China. Liver Int. liv., 00: 1-9. 


\section{إعتلال الكبد كعامل تتبؤى لمعدل وفيات مرضى الكوفيد-19} أثرف عبد العاطى الثناوى عمارة*****، أمين محمود أمين حجازى"، أحمد محمد أبو

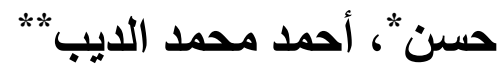

أقسام الباطنة العامة** والأشعة التشخيصية*** والفسيولوجيا الطبية***

كلية الطب، جامعة الأزهر

E-mail: ashrfemara13@gmail.com

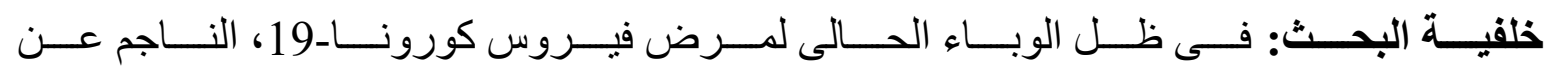

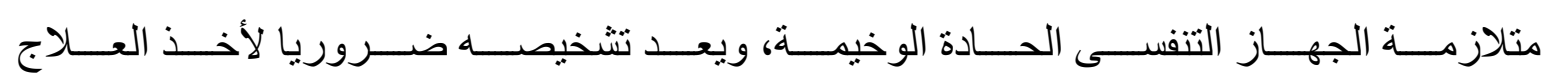

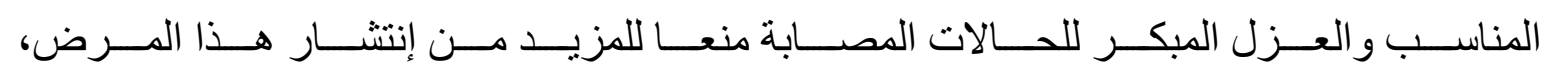
و الذى يكون مصحوبا بشكل كبير بحالات من الأعياء والوفيات.

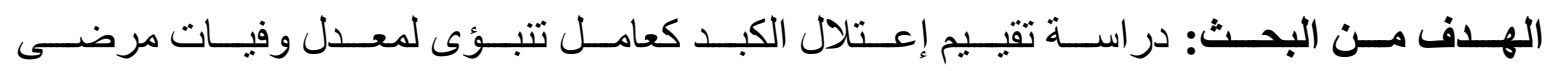

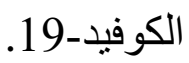

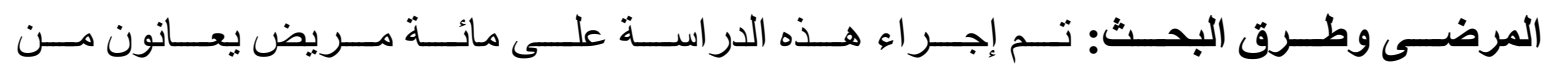

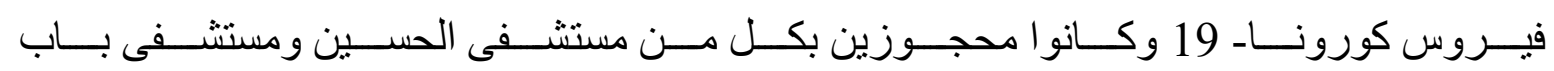

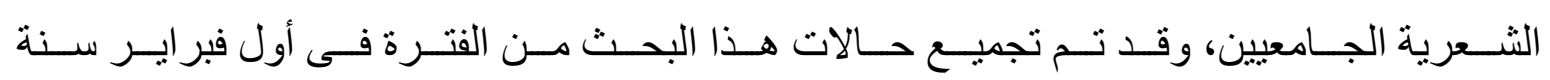

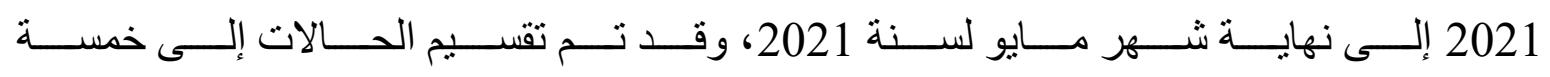

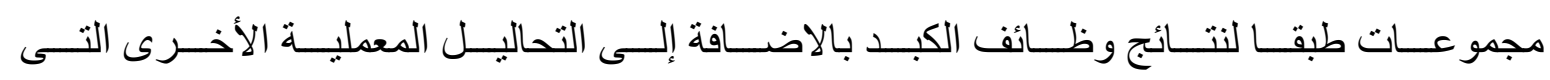

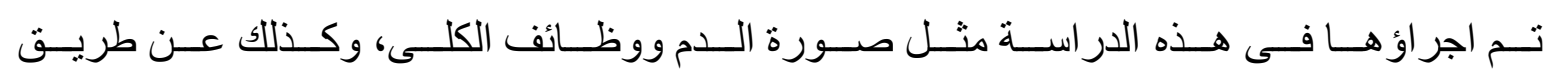
اجر اء الأشعة المقطعية.

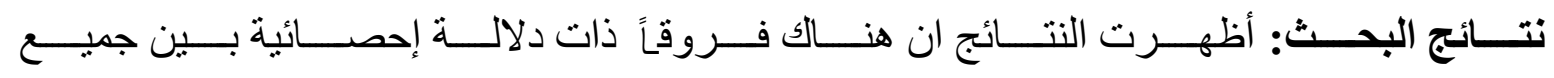

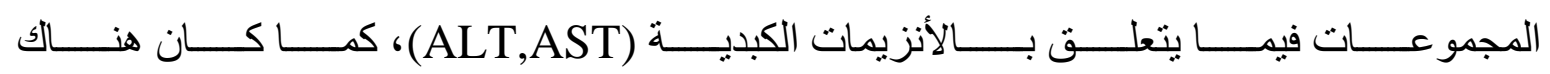

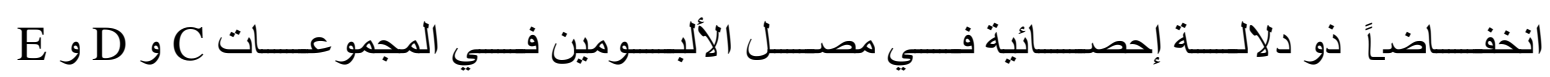

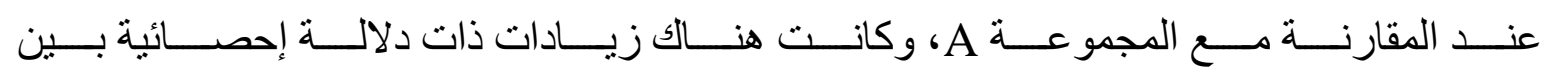

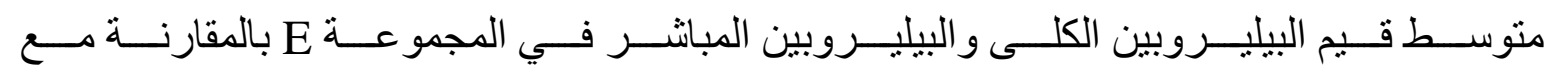

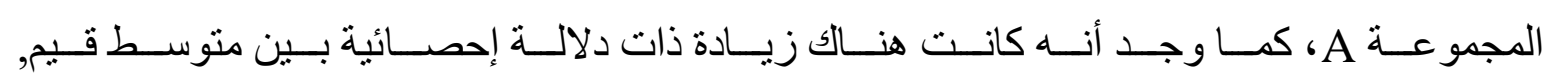




\section{LIVER IMPAIRMENT AS A PREDICTIVE FACTOR FOR MORTALITY... 3161}

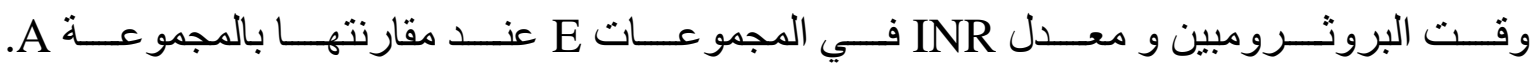

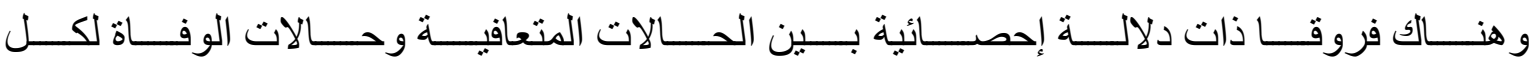

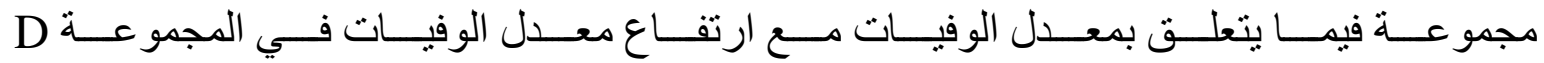

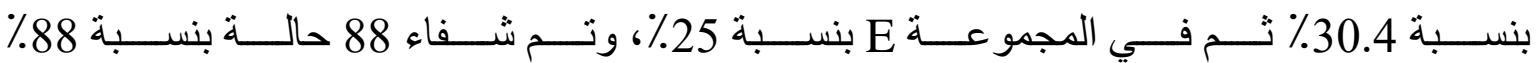
وتوفي 12 حالة بنسبة 12\% في جميع مرضى الكوفيد-19.

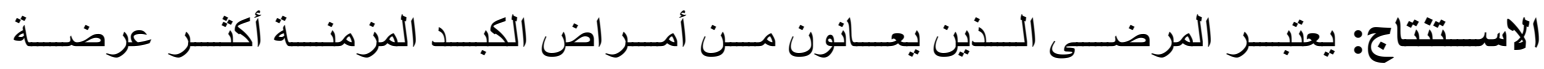

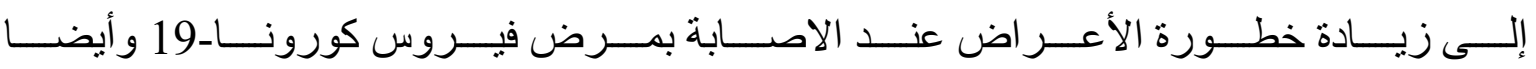

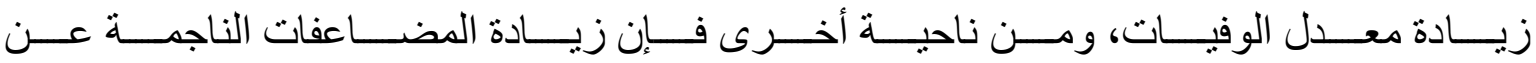

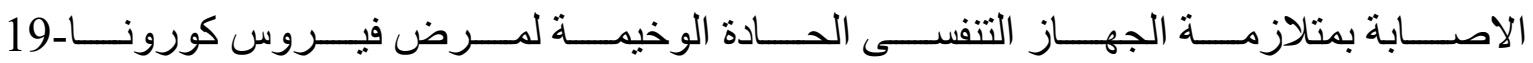
يزيد من نسبة خطورة الإصسابة بالفثل الكبدي.

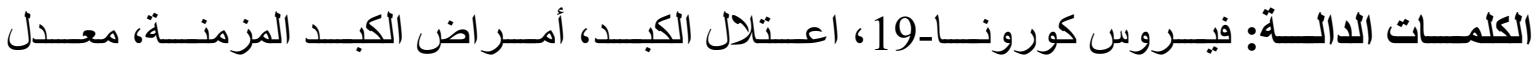
الوفيات. 